\title{
L'incontro col professore
}

\author{
Valeria Della Valle
}

PUBBLICATO: 31 DECEMBER 2020

I 1 titolo del mio intervento, troppo lungo e non adatto all'occasione, dovrebbe essere "Come l'incontro con un professore può cambiare il corso di una vita". Perché l'incontro con Arrigo Castellani ha davvero cambiato il corso della mia vita, e vorrei raccontarlo brevemente, non come fatto privato, ma come esempio dell'importanza del ruolo di chi insegna, e di quello che può rappresentare per la vita degli allievi.

Ho conosciuto Arrigo Castellani nei primi anni del suo insegnamento nella Facoltà di Lettere e Filosofia di Roma, che allora non si chiamava ancora Sapienza. Avevo scelto quella Facoltà non per vocazione o per passione, ma perché nessuna delle altre mi interessava davvero, e soprattutto perché non avevo le capacità per seguire discipline che sentivo lontane e inaccessibili.

Fin dal primo anno di corso avevo avuto la fortuna di poter seguire le lezioni di professori già allora molto famosi: cito almeno Walter Binni, Natalino Sapegno, Giacomo Debenedetti, Giulio Carlo Argan, Ettore Paratore. Professori che incantavano gli studenti che affollavano le aule e seguivano le lezioni in religioso e adorante silenzio. Lezioni indubbiamente suggestive, ma quel modo di presentare e commentare la letteratura e la storia dell'arte suscitava in me molti dubbi. Le interpretazioni e le letture che ascoltavo mi sembravano interessanti, ma molto soggettive, in qualche caso anche fortemente ideologiche. Non mi davano alcuna certezza che in quel passo un poeta volesse alludere davvero a quelle teorie, o che in quel quadro ci fossero riferimenti filosofici di un certo tipo. Nel mio atteggiamento c'era molta ingenuità, ne sono consapevole, ma quelle che cercavo erano certezze, non interpretazioni, teorie, letture personali.

Solo ascoltando le lezioni di Arrigo Castellani trovai quelle certezze: il suo modo di spiegare ogni singolo fenomeno linguistico non era basato su punti di vista, ma sul percorso fatto da ogni parola che il professore commentava per noi, scrivendola alla lavagna. Spiegazioni fatte in modo chiarissimo, nitido, quasi matematico, senza mai aggiungere commenti o impressioni personali, senza alcun compiacimento o esibizione istrionica: la parola spiegata prendeva vita davanti a noi e procedeva dalla sua origine più lontana fino all'esito finale, attraverso tutte le trasformazioni subite, descritte con un metodo che mi conquistò perché era un metodo scientifico, ai miei occhi sicuro e rassicurante.

Aggiungo, come aneddoto, che il rigore e la concentrazione assoluta del professore mentre spiegava i fenomeni linguistici riuscirono a disorientare perfino un gruppo di studenti entrati a interrompere una sua lezione - erano anni politicamente molto caldi -, perché Castellani continuò imperterrito a tracciare sulla lavagna le derivazioni e le trasformazioni fonetiche dei termini, tanto da far allontanare senza polemiche, persa ogni speranza di successo, chi aveva provato a interromperlo.

Cosi, dopo aver dato più esami di storia della lingua italiana, decisi di abbandonare il progetto di laurearmi con una tesi in letteratura italiana, che ormai vedevo come una esercitazione basata su interpretazioni critiche opinabili e su schieramenti dogmatici, e di chiedere invece la tesi di laurea a quel professore elegante, dai modi gentili, direi nobili, che dal primo incontro in un'aula della Facoltà continuava a ricordarmi un ritratto cinquecentesco di scuola toscana. 
Un'altra differenza tra quel professore e gli altri docenti consisteva nel modo di accogliere gli studenti. In quegli anni i professori, soprattutto quelli noti e venerati, erano circondati da schiere di assistenti o aspiranti tali, ed era molto difficile essere ricevuti senza superare sbarramenti e filtri. Per parlare con Arrigo Castellani bastava bussare alla porta verde del suo studio, al terzo piano della Facoltà, nell'Istituto di Filologia moderna, e si era subito accolti dal professore e da Francesco Agostini, il suo giovane assistente, purtroppo mancato prematuramente e tragicamente nel I98I. Voglio ricordare che Francesco Agostini aveva già pubblicato saggi importanti sul volgare perugino e sull'Umbria medievale, e avrebbe continuato di sicuro con successo la carriera universitaria se una serie di drammatiche circostanze non l'avessero interrotta dopo un periodo passato a insegnare l'italiano all'Università Nazionale Somala di Mogadiscio (di quell'esperienza resta il Dizionario somaloitaliano, pubblicato dopo la sua scomparsa).

Proprio grazie alla facilità del contatto col professore e col suo assistente mi fu assegnata subito la tesi: avrei analizzato gli aspetti linguistici delle lettere scritte da mercanti senesi della fine del XIII secolo, i cui originali erano conservati nell'Archivio di Stato di Siena. Andai più volte a Siena a controllare quelle lettere, prive di ambizioni d'arte, ma che avevano ai miei occhi - me ne resi conto più tardi - la stessa grazia e la stessa semplice eleganza della pittura senese delle origini.

Nell'affrontare quella prima prova sperimentai il "metodo Castellani": dovevo sezionare le lettere dei mercanti, catalogando ogni singolo fenomeno sulla base delle nozioni di grammatica storica apprese durante le lezioni. Come guida avrei avuto i quaderni con gli appunti del professore (che conservo ancora), i suoi Nuovi Testi fiorentini del Dugento e, naturalmente, le opere degli studiosi i cui nomi avevo imparato a conoscere da poco: Wilhelm Meyer-Lübke, Gerhard Rohlfs, Alfredo Schiaffini. Tutto era chiaro, mi sembrava di aver trovato le certezze che cercavo.

Durante il lavoro della tesi conobbi un altro aspetto del professore: ogni capitolo veniva letto da Castellani parola per parola, e questo controllo continuava anche durante la pausa estiva, a Quercianella, dove venivo accolta per rivedere le varie parti della tesi. Ricordo che rimanevo colpita dal fatto che Castellani controllasse personalmente ogni forma sui dizionari e sui testi che aveva portato nella casa al mare, collocati in un settore diverso della libreria, rispetto alle collezioni dei gialli e dei libri di fantascienza in edizione originale (questo aspetto del professore mi sorprendeva e me lo rendeva ancora più simpatico). A Quercianella trovavo anche il conforto dell'ospitalità da parte del resto della famiglia, la moglie Ornella Pollidori (che tanti anni dopo avrebbe dedicato la raccolta dei suoi saggi "A Enrico/Arrigo, impareggiabile compagno di vita e di riva") e i tre figli: un ragazzino e due bambine, Leonardo, Elena e Ilaria, che tra di loro parlavano in francese, con i quali condividevo ogni volta un ottimo pranzo. Ricordo con gran piacere l'elegante semplicità di quelle colazioni nel giardino, tra gli alberi, e le conversazioni con il professore e la professoressa, che solo in seguito diventarono per me Arrigo e Ornella; poco lontano c'erano "i bungali", destinati agli ospiti. In quelle occasioni imparai anche ad apprezzare, superato il primo imbarazzo, i silenzi del professore, che parlava solo se aveva qualcosa da dire (altra lezione di vita), e che spesso, durante un silenzio che mi sembrava lunghissimo, continuava a ragionare sull'ultima cosa detta prima dell'interruzione, che fosse una questione fonetica, il dubbio su una pronuncia oppure la parola italiana o italianizzata da scegliere al posto di una straniera. Quando la spedizione a Quercianella era finita e nel dopopranzo avevamo esaminato tutte le schede una per una, il professore mi accompagnava alla stazione, dove mi affidava al capotreno (avevo già più di vent'anni), ricordandogli che, cito le parole di Castellani, "la signorina dovrà scendere a Roma, alla Stazione Termini".

Ho rievocato le spedizioni a Quercianella ma dovrei ricordare anche quelle a Firenze, nella casa di via di Barbacane, di cui non è possibile dimenticare la bellezza e l'atmosfera (i quadri, i preziosi libri 
antichi, il panorama della città dall'alto). I miei non sono solo cari ricordi personali di anni lontani: ogni volta che lasciavo il professore, osservando in treno le numerose correzioni alle mie schede imperfette imparavo come si dovrebbe fare una ricerca ben fatta. Con quale rigore, con quale scrupolo, senza fidarsi mai di dati che non siano stati controllati e ricontrollati nei dizionari e nei testi di riferimento.

In seguito, appena laureata, ho avuto l'occasione di applicare quelle nozioni e quel metodo collaborando, con Luca Serianni, a un programma finanziato dal Consiglio Nazionale delle Ricerche per la trascrizione di documenti in volgare (poi pubblicati molti anni dopo col titolo La prosa italiana delle origini. Testi toscani di carattere pratico). Ricordo questa esperienza per sottolineare un altro aspetto della grande umanità e generosità di Arrigo Castellani: quel volume era dedicato alla memoria di Francesco Agostini.

Ma intanto l'incontro col professore aveva davvero cambiato la mia vita, perché fu lui a presentarmi ad Aldo Duro, che aveva appena avviato i lavori per la redazione di quello che sarebbe diventato il Vocabolario della lingua italiana dell'Istituto della Enciclopedia Italiana Treccani, redazione nella quale nel corso degli anni imparai "che cos'è un vocabolario", per dirla con Migliorini, e soprattutto come si fa un vocabolario. Anche questo lo devo al professore.

Voglio ricordare ancora un aspetto di Castellani: quando partecipai, grazie al suggerimento, all'incoraggiamento e soprattutto all'amicizia di Francesco Agostini al concorso da ricercatrice, dopo che lo ebbi vinto il professore, che ormai da anni non insegnava più a Roma, ma a Firenze, oltre a indirizzarmi verso l'analisi di nuovi testi non letterari, conservati nella Biblioteca Guarnacci di Volterra, mi disse che da quel momento avevo una grande responsabilità: ricordo la sua espressione seria mentre me lo diceva - ci davamo ancora del lei ed ero sempre un po' in soggezione nei suoi confronti - ma so per certo che quel suo ammonimento non l'ho mai dimenticato. In quelle sue parole c'era il rigore non solo scientifico ma etico del professore, per il quale al primo posto, per uno studioso, c'era, c'è, ci deve essere il dovere: il dovere della ricerca scrupolosa e della cura, dell'attenzione nei confronti dei propri studenti e allievi. Non si trattava di una cura, di un'attenzione concorsuale, aspetto che gli era in fondo indifferente, ma dell'attenzione per i risultati del lavoro dei propri allievi: mi riferisco non a me, ovviamente, ma ai quattro allievi che davvero ne hanno continuato e ne continuano tuttora la tradizione e il metodo di lavoro.

Di quel rigore assoluto, di quella disciplina, di quella fedeltà alle proprie idee, senza mai una deflessione, mi resi forse pienamente conto quando nel 2009 - Castellani era morto da cinque anni con Giovanna Frosini, Paola Manni e Luca Serianni curammo i sessantasette studi raccolti nei Nuovi saggi di linguistica e filologia italiana e romanza che vanno dal 1946 al 1976, che Castellani aveva continuato a correggere, postillare e perfezionare fino alla fine. Per prepararne l'edizione ci servimmo anche dei suoi appunti, delle sue note a matita, tracciate nell'inconfondibile e bella grafia sul margine dei contributi. Una volta di più mi resi conto, durante quel lavoro, seguendo la successione degli scritti del professore, non solo del suo rigore, della sua disciplina, della sua coerenza, ma anche dell'ampiezza dei suoi interessi: dal toscano medievale alla terminologia, dalle edizioni di testi antichi al rapporto tra italiano e inglese, dalla storia di singole parole alle questioni di critica testuale.

Il profilo scientifico di Arrigo Castellani è stato tracciato, in altre occasioni, dai suoi allievi e allieve, studiosi e studiose molto più autorevoli di me, e tra poco lo farà Pär Larson. Ma vorrei ancora ricordare solo un episodio, anche per stemperare la commozione di questo momento. Quando con Luca Serianni eravamo ancora studenti, e aspettavamo di essere ricevuti dal professore per la correzione delle nostre tesi di laurea, con la sprovvedutezza dell'età un giorno dissi a Luca: "Il 
professore sa tutto del toscano antico, e noi impareremo da lui, ma Chomsky? Di Noam Chomsky non sappiamo niente!". Questa è rimasta una battuta di lessico familiare scambiata scherzosamente con Luca Serianni, tanti anni fa, seduti su una panca razionalista, nel corridoio dell'Istituto di Filologia Moderna, in anni in cui Chomsky era molto di moda. Oggi so di poter rispondere che poi ho avuto tutto il tempo per informarmi su Chomsky e leggere i suoi scritti, mentre sul nostro professore, su quello che ha scritto, su quello che ci ha lasciato non abbiamo ancora finito di imparare.

Sono certa di poter dire anche a nome di chi ha condiviso la stessa esperienza (Giovanna Frosini, Pär Larson, Paola Manni, Luca Serianni, e idealmente aggiungo Francesco Agostini) che noi, suoi allievi, abbiamo avuto la grande fortuna di incontrare Arrigo Castellani: per noi non un professore, ma il Professore. Il Professore che ha cambiato la mia, le nostre vite, e al quale, per quell'incontro, saremo sempre grati e riconoscenti.

Opere citate per ricordo:

- Francesco Agostini, Il libro di memorie della confraternita di S. Agostino di Perugia (1322-1338), in "Studi linguistici italiani", VII I967-I970, pp. 99-I55; ID, Il volgare perugino negli "Statuti del 1342", in "Studi di filologia italiana", XXVI I968, pp. 9I-I99; ID, Testi trecenteschi di Cittá di Castello e del contado, a cura di Francesco Agostini, Firenze, Accademia della Crusca 1978; Dizionario somaloitaliano, caporedattori Francesco Agostini, Annarita Puglielli, Ciise Moxamed Siyaad, Gangemi Editore, Roma 1985 .

- Arrigo Castellani, Nuovi testi fiorentini del Dugento, Sansoni, Firenze 1952; ID, La prosa italiana delle origini. Testi toscani di carattere pratico, voll. 2 (Facsimili e Trascrizioni) Pàtron, Bologna I982; ID, Nuovi saggi di linguistica e filologia italiana e romanza (1976-2004), a cura di Valeria Della Valle, Giovanna Frosini, Paola Manni, Luca Serianni, Salerno Editrice, Roma 2009.

- Ornella Castellani Pollidori, In riva al fume della lingua. Studi di linguistica e filologia (1961-2002), Salerno Editrice, Roma 2004.

- Valeria Della Valle, Le lettere in volgare di Filippo Belforti vescovo di Volterra (1348-1353), in "Studi linguistici italiani", VIII (I della nuova serie), II, I982, pp. I55-263.

- Aldo Duro (autore e direttore), Vocabolario della lingua italiana, Istituto della Enciclopedia Italiana, Roma i986-94, 5 voll.

- Luca Serianni, Arrigo Castellani (1920-2004), in "Studi linguistici italiani", XXX (IX della IIII serie), I, 2004, pp. 3-I0.

\section{Cita come:}

Valeria Della Valle, L'incontro col professore, "Italiano digitale", 2020, XV, 2020/4 (ottobredicembre)

DOI: $10.35948 / 2532-9006 / 2020.5441$

Copyright 2020 Accademia della Crusca

Pubblicato con licenza creative commons CC BY-NC-ND 